\title{
Transcriptome Analysis Reveals Significant Differences in Gene Expression of Malignant Pheochromocytoma or Paraganglioma
}

\author{
Yong Joon Suh $\mathbb{D}^{1},{ }^{1}$ Jung Ho Park, ${ }^{1}$ Sanchir-Erdene Bilegsaikhan, ${ }^{1}$ and Dong Jin Lee $\mathbb{D}^{2}$ \\ ${ }^{1}$ Department of Breast and Endocrine Surgery, Hallym University Sacred Heart Hospital, Anyang 14068, Republic of Korea \\ ${ }^{2}$ Department of Otolaryngology-Head and Neck Surgery, Hallym University College of Medicine, Seoul 07441, Republic of Korea \\ Correspondence should be addressed to Yong Joon Suh; nicizm@gmail.com
}

Received 12 February 2019; Revised 13 April 2019; Accepted 18 April 2019; Published 8 May 2019

Guest Editor: Giampaolo Papi

Copyright (C) 2019 Yong Joon Suh et al. This is an open access article distributed under the Creative Commons Attribution License, which permits unrestricted use, distribution, and reproduction in any medium, provided the original work is properly cited.

Prediction of malignant behavior of pheochromocytoma (PC) or paraganglioma (PG) is of limited value. The Cancer Genome Atlas (TCGA) and the French 'Cortico et Médullosurrénale: les Tumeurs Endocrines' (COMETE) network in Paris (France) facilitate accurate differentiation of malignant PC/PG based on genetic information. Therefore, the objective of this transcriptome analysis is to identify the prognostic genes underlying the differentiation of malignant PC/PG in the TCGA and COMETE databases. TCGA carries data pertaining to multigenomic analysis of $173 \mathrm{PC} / \mathrm{PG}$ surgical resection samples while the COMETE cohort contains data involving $188 \mathrm{PC} / \mathrm{PG}$ surgical resection samples. Clinical information and mRNA expression datasets were downloaded from TCGA and COMETE databases. Based on eligibility criteria, 58 of 173 PC/PG samples in TCGA and 171 of 188 PC/PG samples collected by the COMETE network were selected. Using Ingenuity Pathway Analysis, the mRNA expression of malignant and benign PC/PG was compared. The 58 samples in TCGA included 11 malignant and 47 benign cases. Among the 171 samples obtained from the COMETE cohort, 19 were malignant and 152 were benign. A comparative analysis of the mRNA expression data of the two databases revealed that $11 \mathrm{up} /$ downregulated pathways involved in malignant PC/PG were related to cancer signaling, metabolic alteration, and prominent mitosis, whereas 6 upregulated genes and 1 downregulated gene were significantly enriched in the functional annotation pathways. The TCGA and COMETE databases showed differences in mRNA expression associated with malignant and benign PC/PG. Improved recognition of prognostic genes facilitates the diagnosis and treatment of PC/PG.

\section{Introduction}

Pheochromocytoma (PC) is a catecholamine-secreting neuroendocrine neoplasm originating in the adrenal medulla [1]. Paraganglioma (PG) is a catecholamine-producing neuroendocrine neoplasm developing in the extra-adrenal chromaffin tissue of sympathetic ganglia. Nearly $15-20 \%$ of PC/PG originated in extra-adrenal chromaffin tissues whereas $80-85 \%$ of $\mathrm{PC} / \mathrm{PG}$ develops from adrenal medulla. The annual incidence of $\mathrm{PC} / \mathrm{PG}$ varies between 2 and 8 per million. In the population, the prevalence of PC/PG ranges from 1:6,500 to $1: 2,500$, respectively $[2,3]$. Clinical manifestations include hypertension, tachycardia, headache, diaphoresis, and anxiety [4].
Diagnostic tests for PC/PG include imaging, biochemical evaluation, and histopathology, in addition to genetic testing [5-7]. Patients with PC/PG are managed via surgery, medical treatment, chemotherapy, targeted radiation therapy using ${ }^{131}$ I-MIBG, embolization, cryoablation, targeted molecular therapy, and radiofrequency ablation [8]. Due to the diagnostic uncertainty, management usually entails vigilant monitoring for metastasis in PC/PG.

The tumor conforms to "the rule of $10 \mathrm{~s}$ " and $10 \%$ of $\mathrm{PC} / \mathrm{PG}$ is considered malignant $[9,10]$. However, the malignancy rate exceeds $10 \%$ in patients with extra-adrenal disease [11]. Malignant PC/PG is associated with a 5-year survival rate of around $50 \%[12,13]$. The patients' long-term survival has yet to be improved [14]. 
Histological analysis cannot be used to predict the malignant or benign behavior of PC/PG [15]. According to the 8th edition of the American Joint Committee on Cancer (AJCC) staging system, distant metastasis is the only distinctive feature of malignant PC/PG. Therefore, this diagnostic limitation restricts therapeutic planning. Accurate diagnosis is directly linked to successful management. Institutions have struggled to define molecular markers for malignant PC/PG $[16,17]$. Studies to discover robust predictors of malignancy are still ongoing $[18,19]$.

Recent molecular data obtained from The Cancer Genome Atlas (TCGA) and the French 'Cortico et Médullosurrénale: les Tumeurs Endocrines' (COMETE) cohort are available in the public domain [20-22]. The databases describe tumors including mRNA expression and clinical information. The TCGA and COMETE databases may be used for transcriptome analysis of malignant PC/PG. The aim of the present study was to identify potential biomarkers based on the mRNA expression profile of TCGA and COMETE databases for diagnosis of malignant PC/PG.

\section{Materials and Methods}

2.1. Study Design. Accessible data obtained from TCGA and COMETE cohort were used to compare malignant and benign PC/PG cases. The TCGA data contains multigenomic analysis of $173 \mathrm{PC} / \mathrm{PG}$ surgically resected samples. The initial pathological diagnosis was conducted from 1988 to 2013. Clinical information and gene expression dataset (20,531 genes) of RNA-sequencing mRNA Fragments Per Kilobase Million (FPKM) were downloaded from the TCGA database. The dataset was assembled into a table. Only samples with TCGA type code 01 (primary solid tumor) were included (Figure 1). Benign samples were included only if the followup exceeded two years. However, malignant samples were included even if they were metastasized within two years. Aggressive samples with local invasion or metastatic lymph nodes were excluded due to uncertain behavior. A total of 58 samples met the inclusion criteria. The $58 \mathrm{PC} / \mathrm{PG}$ samples included 11 malignant and 47 benign cases. Clinical characteristics and mRNA expression were reorganized according to tumor behavior and compared with each other. The COMETE cohort carried multiomic analytical data pertaining to $188 \mathrm{PC} / \mathrm{PG}$ surgically resected samples from the French COMETE Network. Cases were recruited from 1993 to 2008. Clinical information and expression dataset (39,534 probes) of mRNA transcripts were downloaded from the COMETE cohort. Only primary tumor samples were included (Figure 2). A total of 171 samples contained accessible genomic data and corresponding clinical information. The 171 samples included 19 malignant and 152 benign cases. Clinical characteristics and mRNA expression were reorganized according to behavior and compared. The gene expression was interpreted by extracting differentially expressed genes (DEGs) from TCGA and COMETE cohort. Commonly enriched genes were searched via Ingenuity Pathway Analysis (IPA). The identified genes were validated using the Dutch cohort (GSE67066) [23].
2.2. Definition. Metastasis was defined as the presence of chromaffin tissue at nonchromaffin sites distant from the primary tumor. Malignant or benign behavior was used to define metastasis or lack thereof, respectively. A zero value was filtered for the analysis of mRNA. To identify DEGs, a false discovery rate $<0.05$ and a $\log _{2}$ fold change $\geq 1.5$ were set as the threshold values. The functional enrichment of genes was analyzed based on Gene Ontology.

2.3. Statistics. Malignant and benign PC/PG groups were compared using Chi-squared test or Fisher's exact test for categorical data and Student's t-test or Mann-Whitney $U$ test for continuous data without normal distribution. In twotailed tests, a $p$ value below 0.05 was considered statistical significant. Statistical analysis was performed using R 3.4.1 (R Foundation for Statistical Computing, Vienna, Austria), GSEA 2.1.0 (Broad Institute, MA, USA), and IPA (Ingenuity Systems, CA, USA). The overall survival rate was estimated according to the degree of gene expression using the UALCAN platform [24].

\section{Results}

3.1. Clinical Characteristics. A total of 58 patients with PC/PG from TCGA were included in the current study. They included 11 patients with metastasis assigned to the malignant PC/PG group. Among 171 patients with PC/PG derived from COMETE cohort, there were 19 patients with malignant PC/PG. TCGA and COMETE cohort showed no age or gender difference in malignant and benign manifestations (Table 1). The mean follow-up periods were $2460.2 \pm 2658.9$ days and $1617.6 \pm 867.7$ days in malignant and benign $P C / P G$, respectively $(p=0.320)$. The proportion of cases diagnosed with paraganglioma was significantly higher among cases of malignant PC/PG $(p<0.001)$. The size was also significantly greater in malignant PC/PG $(p<0.001)$. The optimal cutoff value was calculated to identify malignancy. According to the ROC curves, the highest accuracy was obtained with a size of $54.5 \mathrm{~mm}$ (AUC $=0.778$, sensitivity $=66.7 \%$, specificity $=61.6 \%$, and $p<0.001$ ) (Figure 3 ). Dopamine secretion was more frequent in malignant $\mathrm{PC} / \mathrm{PG}$, whereas metanephrine secretion was only detected in benign cases of PC/PG (Table 2).

3.2. $m R N A$ Analysis. In TCGA, 6,056 out of 20,531 genes were excluded because at least one sample scored zero value. Based on a comparative analysis of 14,475 genes, 367 upregulated genes were identified while 282 genes were downregulated. A total of 39,534 probes were used to analyze COMETE cohort. Results showed an upregulation of 558 probes and downregulation of 1,132 probes. In both data, the quality control was performed for each gene or probe. The gene expression was analyzed in various ways. Functional annotation analysis was used for gene set enrichment (Figure 4). The top 50 features identified in Gene Set Enrichment Analysis are listed in Supplemental Table 1. Commonly enriched pathways in malignant PC/PG were linked to significant mitosis, metabolic alteration, and cancer signaling (Figure 5). Hierarchical clustering analysis was 
TABLE 1: Clinical demographics of malignant and benign PC/PG cases obtained from the TCGA database and the COMETE cohort.

\begin{tabular}{|c|c|c|c|}
\hline Characteristic & Malignant $(\mathrm{n}=30)$ & Benign $(n=199)$ & $p$ value \\
\hline Age (years), mean \pm SD & $38.9 \pm 14.4$ & $43.2 \pm 15.7$ & 0.140 \\
\hline Gender, n (\%) & 30 & 199 & 0.432 \\
\hline Male & $15(50.0)$ & $83(51.1)$ & \\
\hline Female & $15(50.0)$ & $116(48.9)$ & \\
\hline Race, n (\%) & 11 & 46 & 0.497 \\
\hline White & $10(90.9)$ & $36(78.2)$ & \\
\hline African-American & $1(9.1)$ & $5(10.9)$ & \\
\hline Asia & $0(0)$ & $5(10.9)$ & \\
\hline Laterality, n (\%) & 6 & 41 & 0.749 \\
\hline Right & $2(33.3)$ & $19(46.4)$ & \\
\hline Left & $4(66.7)$ & $21(51.2)$ & \\
\hline Bilaterality & $0(0)$ & $1(2.4)$ & \\
\hline Diagnosis, n (\%) & 30 & 199 & $<0.001$ \\
\hline PC & $15(50.0)$ & $177(87.2)$ & \\
\hline PG & $15(50.0)$ & $22(12.8)$ & \\
\hline Size $(\mathrm{mm})$, mean $\pm \mathrm{SD}$ & $73.4 \pm 28.1$ & $46.8 \pm 20.2$ & $<0.001$ \\
\hline Follow-up (days), mean \pm SD & $2460.2 \pm 2658.9$ & $1617.6 \pm 867.7$ & 0.320 \\
\hline
\end{tabular}

TABLE 2: Catecholamine secretion by malignant and benign PC/PG cases derived from TCGA.

\begin{tabular}{lccr}
\hline Characteristic & Malignant $(\mathrm{n}=9)$ & Benign $(\mathrm{n}=40)$ & $p$ value \\
\hline Biochemical testing & & & 34 \\
Normetanephrine & 6 & 24 & 0.336 \\
Norepinephrine & 5 & 15 & 1.000 \\
Epinephrine & 1 & 21 & 0.238 \\
Metanephrine & 0 & 0 & 0.006 \\
Methoxytyramine & 1 & 5 & 0.184 \\
Dopamine & 4 & 0.046 \\
\hline
\end{tabular}

PC: pheochromocytoma; PG: paraganglioma.

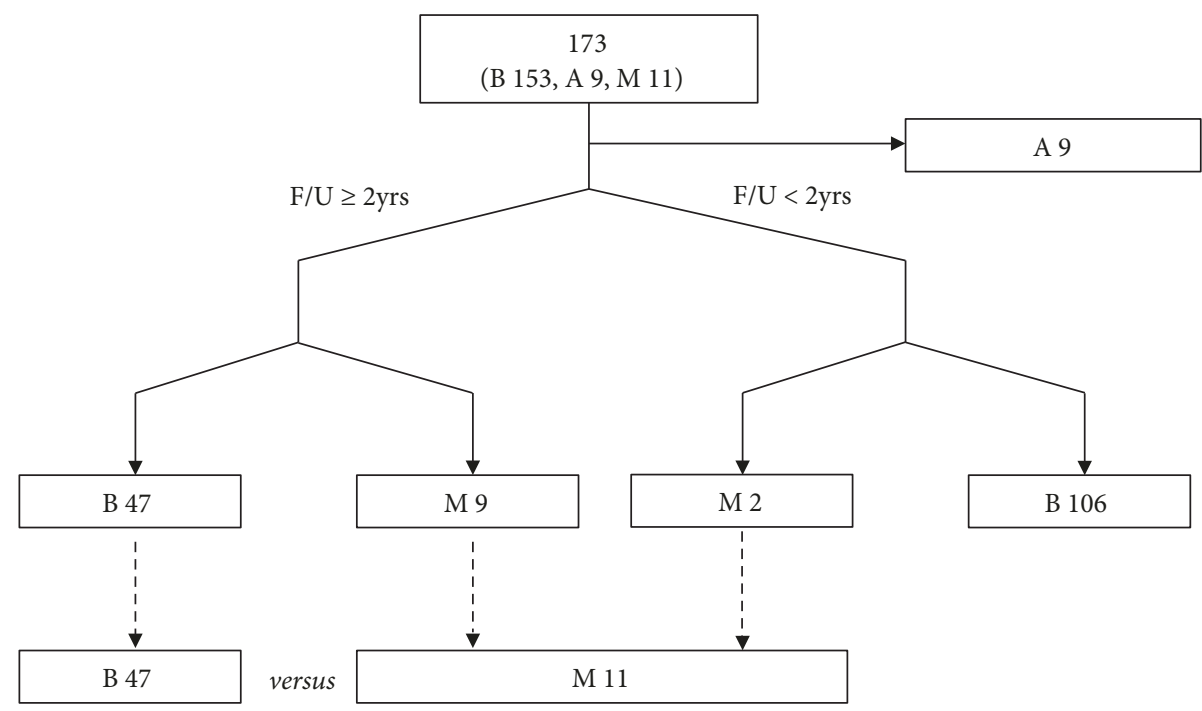

FIGURE 1: Flow diagram outlining enrollment protocol in TCGA. PC: pheochromocytoma; PG: paraganglioma; M: malignant; A: aggressive; B: benign; F/U: follow-up. 


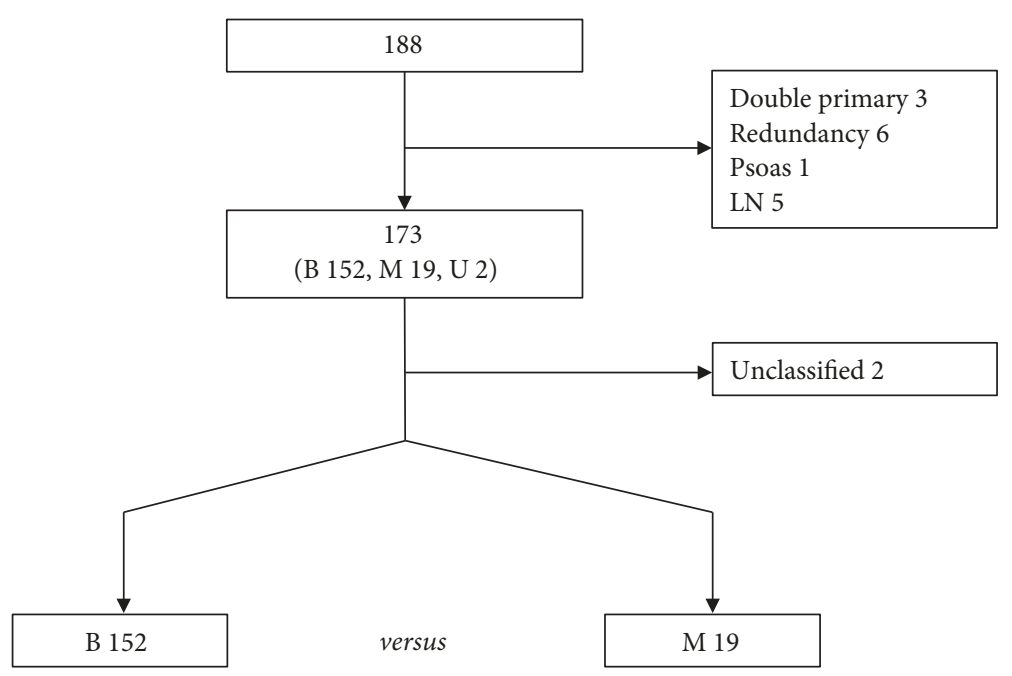

FIGURE 2: Flow diagram outlining the enrollment protocol in COMETE cohort. PC: pheochromocytoma; PG: paraganglioma; M: malignant; B: benign; U: unclassified; LN: lymph node.

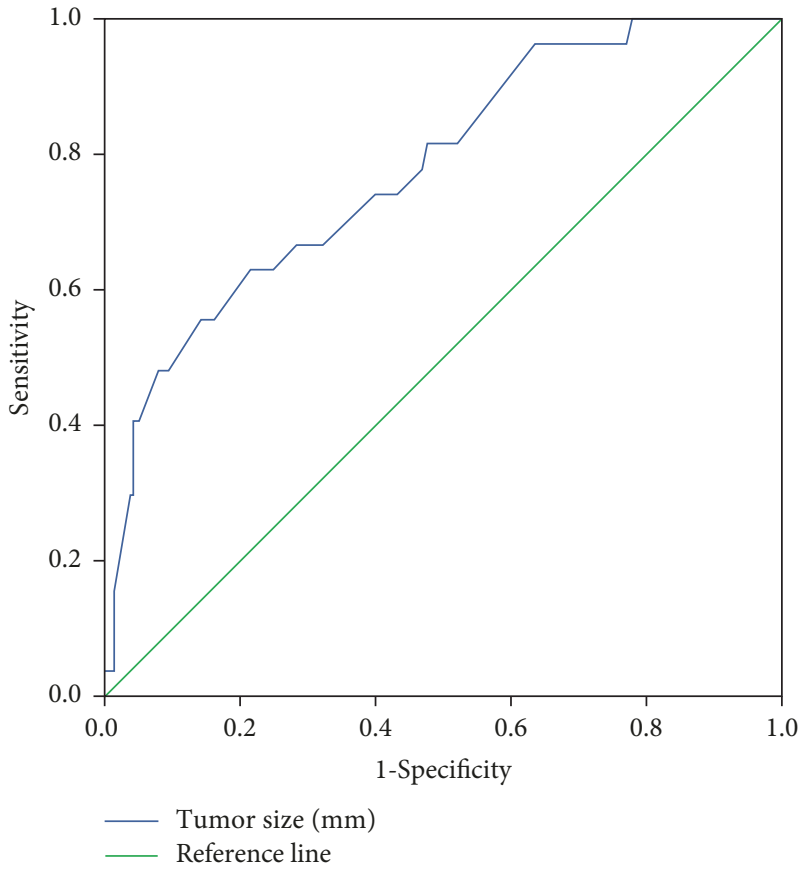

FIGURE 3: According to the ROC curves, the size of $54.5 \mathrm{~mm}$ yielded the highest accuracy in TCGA and COMETE cohort $(\mathrm{AUC}=0.778$, sensitivity $=66.7 \%$, specificity $=61.6 \%$, and $p<0.001)$. ROC: receiver operating characteristic; AUC: area under the curve.

performed by Euclidean distance and complete linkage. The common up/downregulated differentially expressed genes (DEGs) were extracted from TCGA and COMETE cohort (Supplemental Table 2). The 11 up/downregulated pathways harbored over/underexpressed genes (Table 3). The cyclin and cell cycle regulation as well as the dopamine receptor signaling representing pathways in TCGA and COMETE cohort were displayed in Figure 6. The seven common genes identified were validated in the Dutch cohort (GSE67066). As potential biomarkers, the seven genes in the current study are presented in bold (Supplemental Table 3). Among the seven common genes identified, the overall survival rate in TCGA was significantly correlated with the expression of four genes (TOP2A, ESPL1, CDK1, and TYMS) (Figure 7).

\section{Discussion}

Currently few reliable histopathological criteria predict malignant behavior in PC/PG. Studies to date reported prognostic factors for malignant $\mathrm{PC} / \mathrm{PG}$ including older age, greater tumor size, extra-adrenal location, elevated dopamine, and synchronous metastasis $[13,14,19,25-$ 27]. In the present study, the possible clinical risk factors included dopamine secretion, PG, and greater tumor size. These observations were generally consistent with previous studies. Differences in genomic expression of malignant PC/PG were investigated using data derived from TCGA and COMETE cohort to predict the clinical prognosis. The 11 up/downregulated pathways in malignant PC/PG were significantly associated with the clinical phenotype of increased tumor size and dopamine secretion. Six upregulated and one downregulated genes were significantly enriched in functional annotation pathways. In PC/PG transformed to malignant types, cellular or nuclear proliferation, signaling network, and metabolic changes were essential processes linked to cancer progression. Among the seven common genes, four genes were considerably correlated with overall survival rate.

Grading for adrenal pheochromocytoma and paraganglioma (GAPP) and pheochromocytoma of the adrenal gland scaled score (PASS) have been developed to predict malignancy based on histopathology [28-30]. These two risk stratification systems show several common features, including high cellularity, tumor necrosis, vascular or capsular invasion, and large nest. The recurrent themes carry implied validity. However, these systems are limited intrinsically or due to the absence of consistent validation $[28,30]$. 


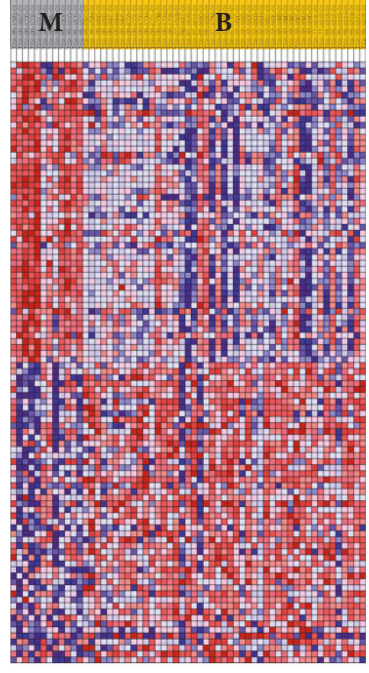

(a)

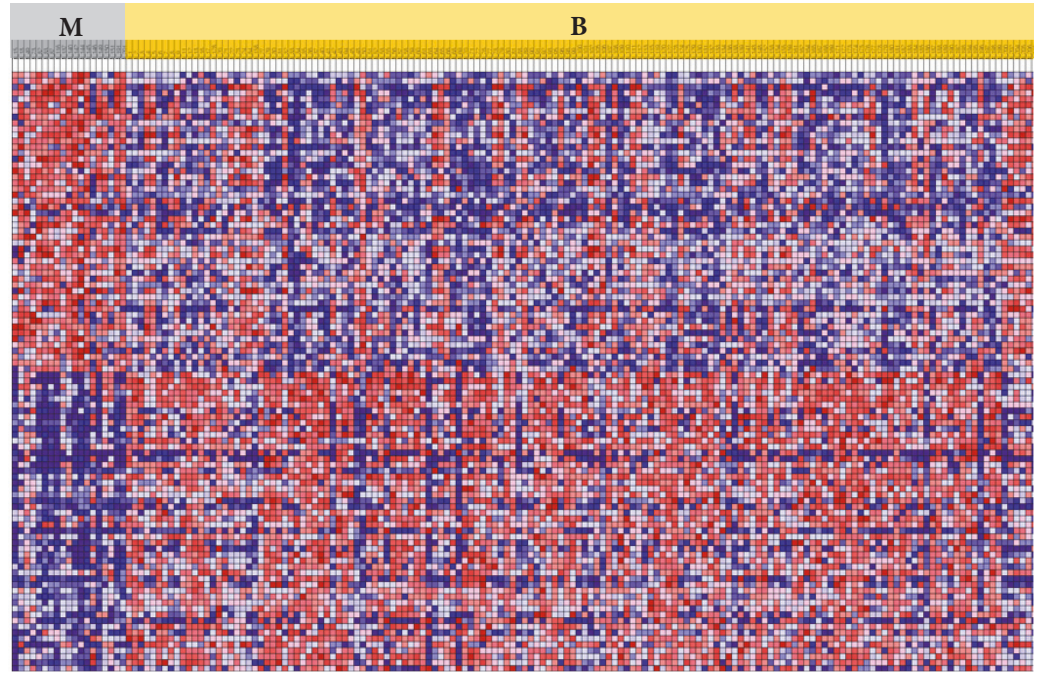

(b)

FIGURE 4: Gene Set Enrichment Analysis showing the heat map of top 50 features for each phenotype based on metastasis of PC/PG cases in (a) TCGA and (b) COMETE cohort. PC: pheochromocytoma; PG: paraganglioma; M: malignant; B: benign.

TABLE 3: Ingenuity Canonical Pathways and the corresponding genes in the transcriptome analysis differentiating malignant PC/PG cases in TCGA and COMETE cohort.

\begin{tabular}{|c|c|c|}
\hline \multirow{2}{*}{ Ingenuity Canonical Pathways } & \multicolumn{2}{|c|}{ Regulation } \\
\hline & $U p$ & Down \\
\hline Cyclins and Cell Cycle Regulation & CCNA2, CDK1 & \\
\hline Dopamine-DARPP32 Feedback in cAMP Signaling & & $P R K A C B$ \\
\hline Mitotic Roles of Polo-Like Kinase & CDK1, ESPL1 & \\
\hline Cell Cycle Control of Chromosomal Replication & CDK1, CDT1, TOP2A & \\
\hline Cardiac $\beta$-adrenergic Signaling & & PRKACB \\
\hline Dopamine Receptor Signaling & & PRKACB \\
\hline Role of CHK Proteins in Cell Cycle Checkpoint Control & $C D K 1$ & \\
\hline Pyrimidine Deoxyribonucleotides De Novo Biosynthesis I & TYMS & \\
\hline Salvage Pathways of Pyrimidine Ribonucleotides & $C D K 1$ & \\
\hline Protein Kinase A Signaling & & PRKACB \\
\hline DNA damage-induced 14-3-3 $\sigma$ Signaling & $C D K 1$ & \\
\hline
\end{tabular}

PC: pheochromocytoma; PG: paraganglioma.

In one study, 58 pheochromocytoma samples were analyzed to distinguish malignant samples [31]. Based on lymph node or distant metastasis, 13 samples were classified as malignant. Genome-wide expression profiling was used to select 10 genes among 109 DEGs which were selected. The present study had a similar focus. However, the current study was designed in response to the challenges documented in the 8th edition of the AJCC staging system. Malignant tumors may not be associated with local invasion or locoregional lymph node metastases. Therefore, a stricter definition of malignancy was used to classify patients with $\mathrm{PC} / \mathrm{PG}$. Data derived from the two public databases were used for consistency. Even in functionally enriched pathways, each cascade was investigated for common genes. The 11 functional pathways were presumably transformed to malignancy, and included six upregulated and one downregulated genes.
The current results were based on accumulated biological information. These genes were validated in TCGA and COMETE cohort. In other studies, the tumorigenesis of PC/PG was explained via alteration in the three representative molecular signaling pathways including pseudohypoxia signaling, kinase signaling, and WNT signaling [18, 20, 21, 32,33 . Activation of effector molecules in pseudohypoxia signaling is triggered by genetic mutations involving the degradation of hypoxia-inducible factor $1 / 2 \alpha$ or Krebs cycle function, such as succinate dehydrogenase genes (SDHx), $V H L$, or EPAS1 [34]. These changes suggest increased levels of angiogenesis and enable hematogenous dissemination [35, 36]. Hyperactive kinase signaling is induced by mutations involving genes associated with mitogen-activated protein kinase, such as tumor suppressor genes (NF1, TMEM127, $M A X$, and $K I F 1 B \beta$ ), RET, or HRAS, which promoted growth 


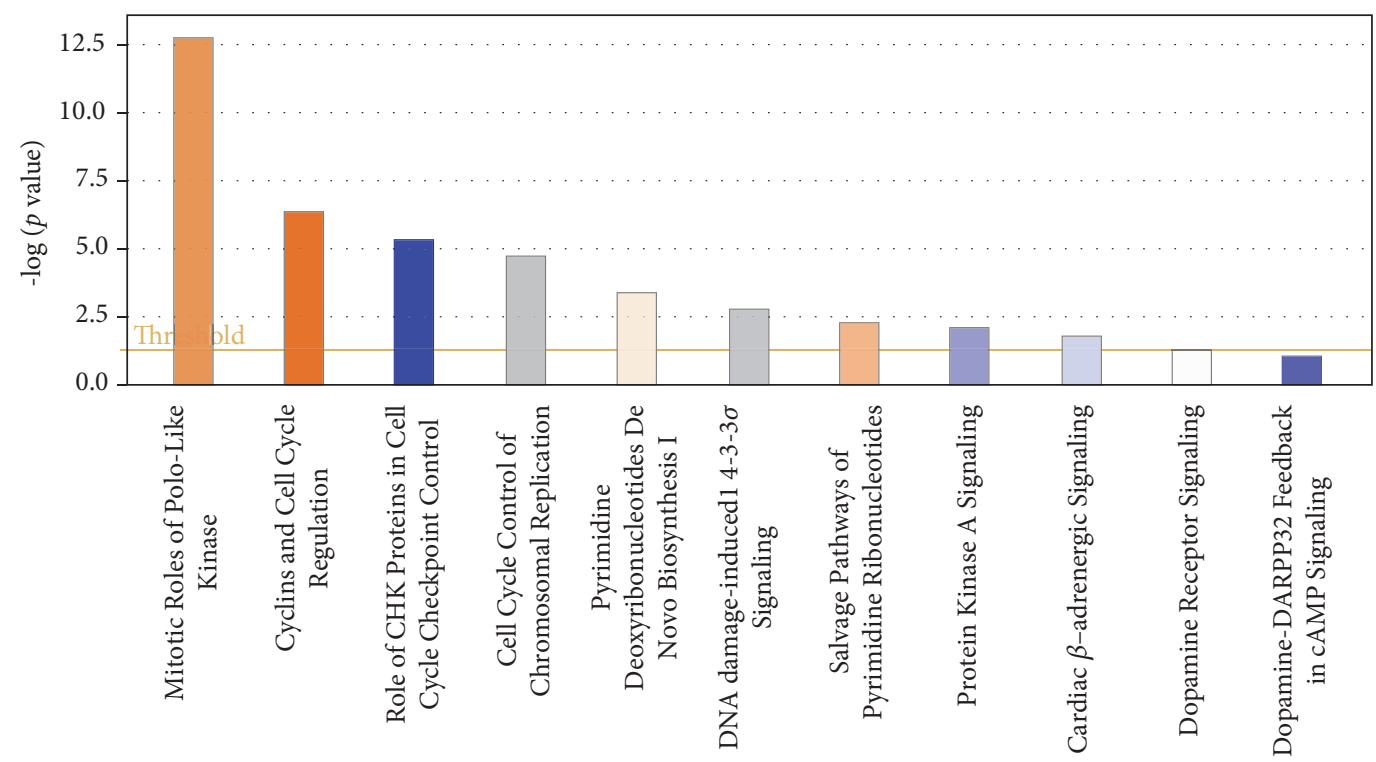

positive $\mathrm{z}$-score

Z-score $=0$

negative $\mathrm{z}$-score

no activity pattern available

(a)

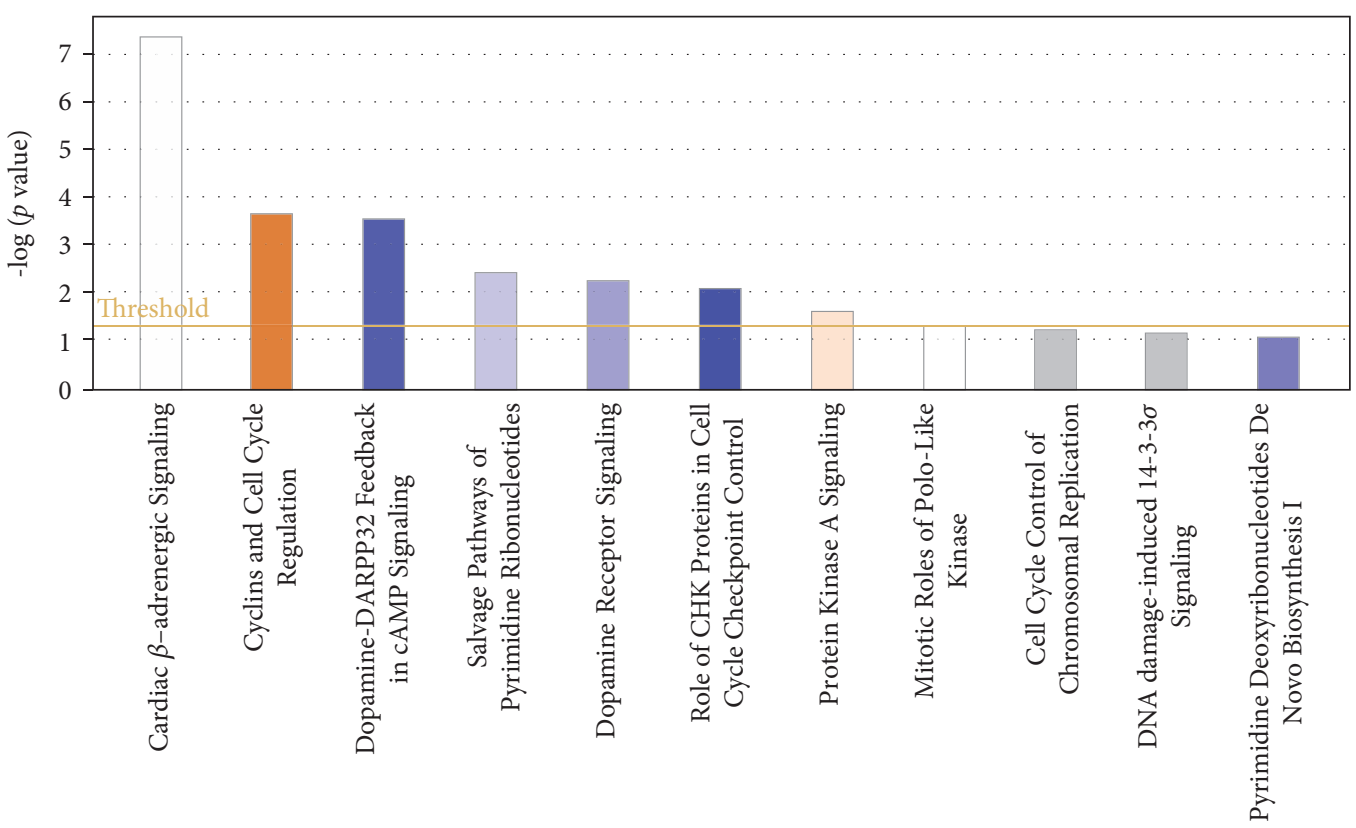

positive z-score

Z-score $=0$

negative $\mathrm{z}$-score

no activity pattern available

(b)

FIGURE 5: Ingenuity Canonical Analysis showing 11 pathways harboring the corresponding genes in the transcriptome analysis to differentiate malignant PC/PG in (a) TCGA and (b) COMETE cohort. PC: pheochromocytoma; PG: paraganglioma. 


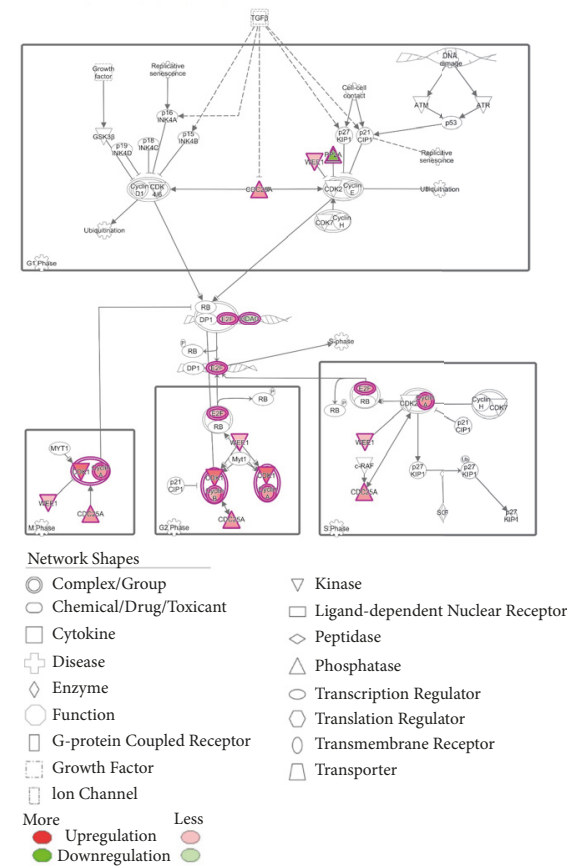

(a)

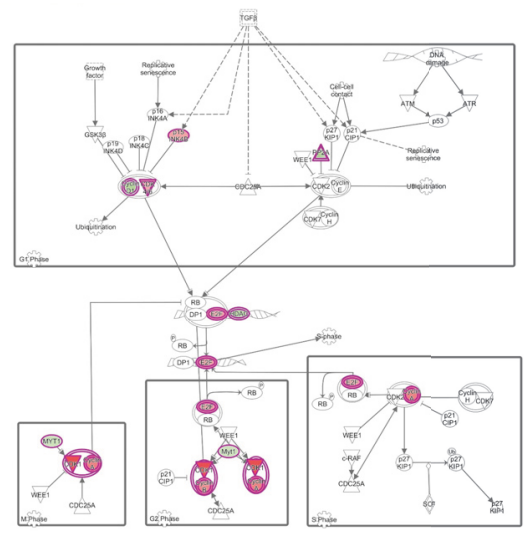

Network Shapes

(2) Complex/Group

$\nabla$ Kinase

Chemical/Drug/Toxicant

$\square$ Cytokine

Disease

$\diamond$ Peptidase

$\checkmark$ Enzyme

G-protein Coupled Receptor

Transcription Regulator

Translation Regulator

Growth Factor

Transmembrane Receptor

lon Channel

More Upregulation Les

$\square$ Transporter

Upregulation

FIGURE 6: Continued.

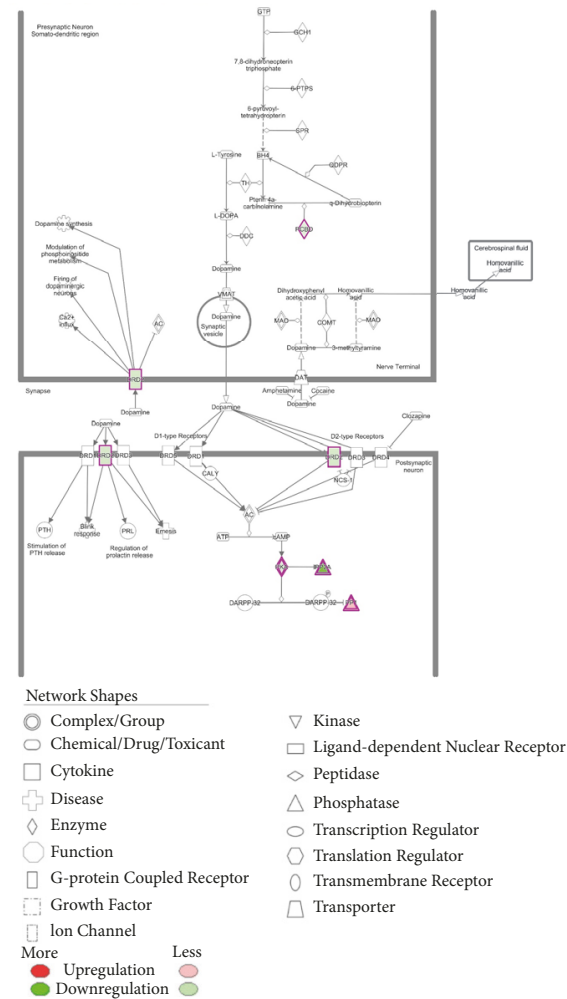

(c)

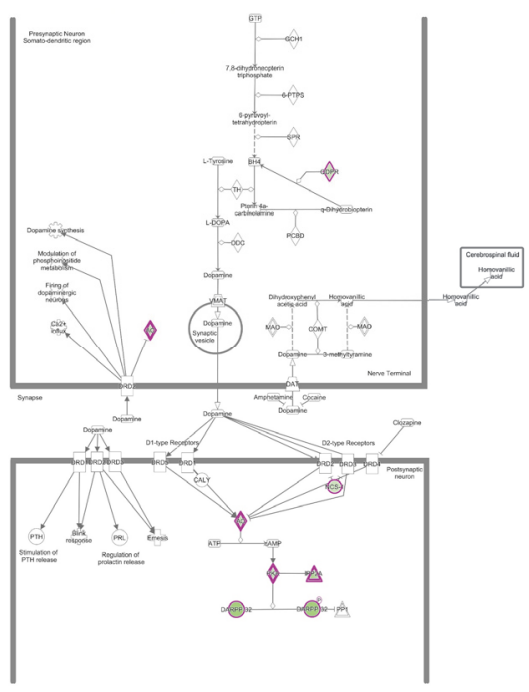

Network Shapes

Complex/Group Kinase

$\checkmark$ Chemical/Drug/Toxicant $\square$ Ligand-dependent Nuclear Receptor

$\square$ Cytokine $\quad \diamond$ Peptidase

$\triangle$ Disease Phosphatase

$\diamond$ Enzyme $\quad$ Transcription Regulator

Function Translation Regulator

$\square$ G-protein Coupled Receptor O Transmembrane Receptor

Growth Factor

lon Channel

$\square$ Transporter

Uore Less

(d)

FIGURE 6: Up/downregulated genes in enriched pathways: cyclins and cell cycle of (a) TCGA and (b) COMETE cohort and dopaminergic synapse of (c) TCGA and (d) COMETE cohort. 


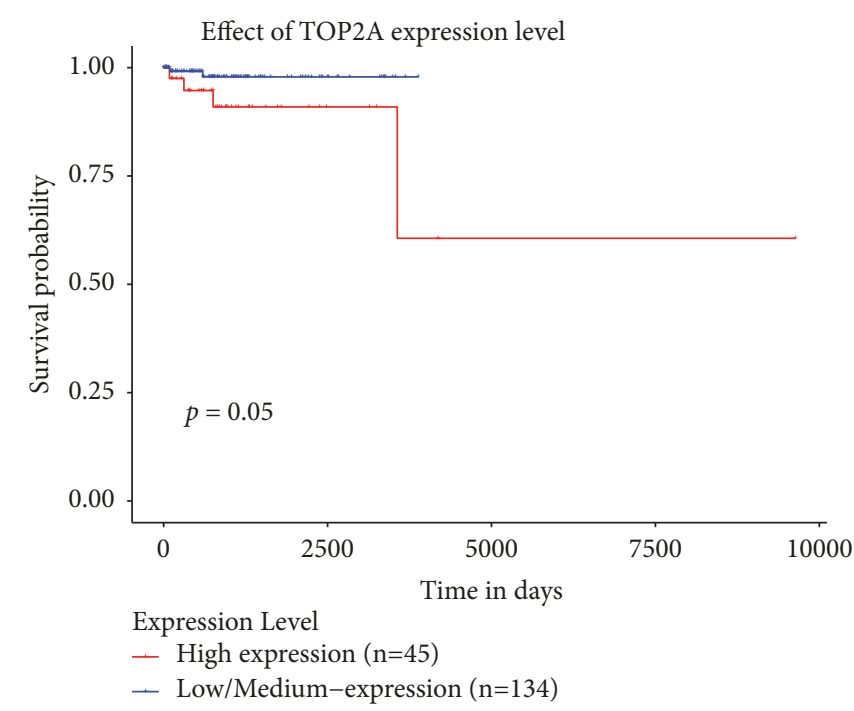

(a)

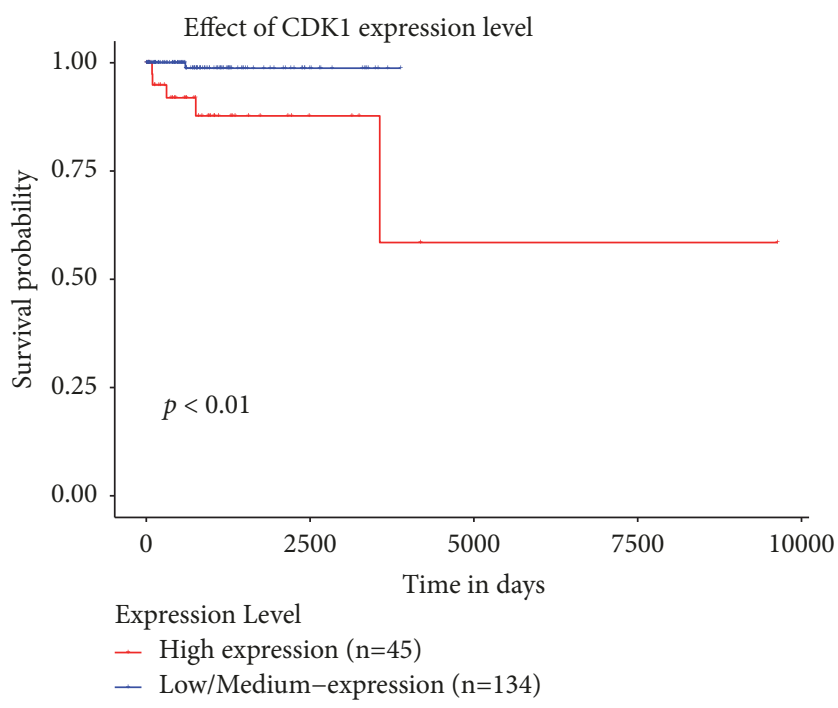

(c)

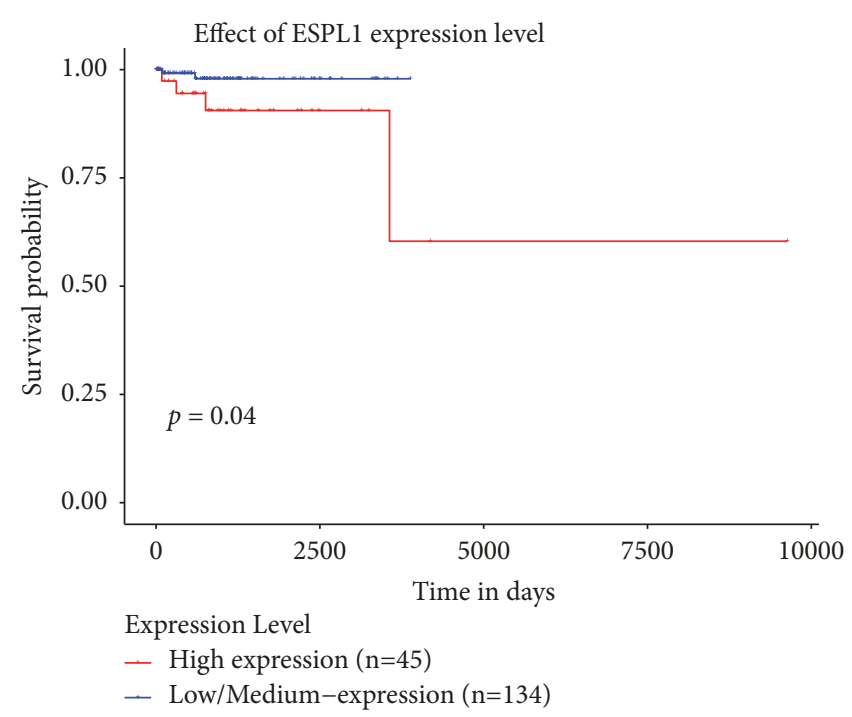

(b)

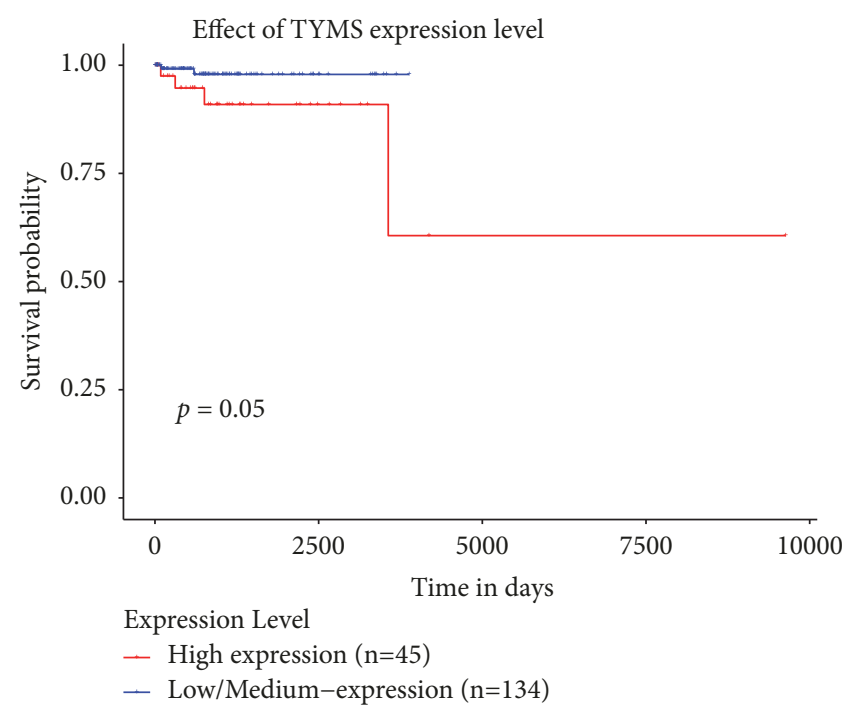

(d)

FIGURE 7: When the identified genes were analyzed in UALCAN, overall survival rate in TCGA was considerably significantly with four genes: (a) TOP2A, (b) ESPL1, (c) CDK1, and (d) TYMS.

independence of extracellular signals [35, 37, 38]. Additionally, upregulated $W N T$ signaling has been recently reported in genetic alterations of MAML3 and CSDE1 [20].

In the present study, DEGs were enriched in 11 presumptive pathways that interacted closely with the three known molecular pathways mentioned above. Further, the 11 presumptive pathways mediated malignant transformation, including cellular or nuclear proliferation, signaling, and metabolic changes predicting cancer progression. However, whether these pathways directly mediated metastasis or the by-product circuits remains to be investigated.

Among the 11 pathways, six common genes (TOP2A, ESPL1, CDK1, TYMS, CDT1, and CCNA2) were differentially overexpressed, and one common gene (PRKACB) was underexpressed in malignant PC/PG. These genes play a role in cell cycle, cell signaling, and tumor metabolism of malignant
PC/PG as well as in other cancers. These six overexpressed genes play a critical role in signaling pathways or cell proliferation, which can increase the tumor size in malignant PC/PG as shown in Table 1. The underexpressed gene was correlated with signaling pathways or metabolic changes, which may lead to differential catecholamine secretion as shown in Table 2. Abnormal TOP2A overexpression leads to chromosomal instability. ESPL1 plays a pivotal role in chromatid separation. The overexpression of CDK1, TYMS, $C D T 1$, and CCNA2 and the underexpression of $P R K A C B$ were associated with tumorigenesis, defective cell signaling, or aberrant metabolism in other cancers [39]. These genes served as prognostic genes for malignant PC/PG in this study.

Germline mutations have been reported in $20-41 \%$ cases of PC/PG [3]. Comprehensive analyses have implicated germline mutations involving $S D H B, F H, M A X$, and 
SLC25A11 in the origin and development of malignant $\mathrm{PC} / \mathrm{PG}$ [40-43]. It is generally recognized that PC/PG carrying germline mutations of $S D H B$ show a higher rate of metastasis [44]. The SDHB mutation in Krebs cycle impairs glucose metabolism, leading to inhibition of 2-oxoglutaratedependent histone and DNA demethylase enzymes. The mutation rate and the spectrum of malignant $\mathrm{PC} / \mathrm{PG}$ were not comparable in these different cohorts. The germline mutation of $S D H B$ was significantly susceptible to malignancy in TCGA and COMETE cohort $(p=0.0049$ and $p<0.001$, respectively). Mutations involving $S D H x$ or $F H$ lead to DNA hypermethylation, explaining both the tumor-suppressive role of these genes and the phenotypic characteristics [45]. In particular, the malignancy of $S D H B$ mutation is attributed to severe epigenetic silencing of genes involved in cell differentiation and epithelial-to-mesenchymal transition. $R D B P$ hypermethylation may alter transcriptional networks involving apoptosis, invasion, and maintenance of DNA integrity [46].

To the best of our knowledge, this study is the first transcriptome analysis identifying prognostic genes for malignant PC/PG defined in the 8th edition of the AJCC staging system. We identified potential pathways leading to malignant transformation of $\mathrm{PC} / \mathrm{PG}$ and subsequently up/downregulated genes in malignant PC/PG. Specific genes in the current study may be used for the development of gene expression classifier [47]. The development of gene expression classifier is expected to improve the diagnostic accuracy and treatment decision. Novel molecular therapeutics can be developed based on the results of the current study.

The current study has a few limitations. First, no comprehensive analysis of microRNA, DNA methylation, copynumber variation, mutation, or protein expression was carried out to establish the complete signature of malignant $\mathrm{PC} / \mathrm{PG}$. Second, only two proven databases were used in the present study. Identification of databases for genomic analysis is difficult because PC/PG is a rare disease. Third, as a general limitation of public data analysis, the present study was based on limited data provided.

In conclusion, data from the TCGA database and the COMETE cohort showed differences in mRNA expression between malignant and benign $\mathrm{PC} / \mathrm{PG}$. Improved recognition of prognostic genes based on our analyses will facilitate appropriate diagnosis and treatment of malignant $\mathrm{PC} / \mathrm{PG}$.

\section{Data Availability}

The data used to support the findings of this study are available from the corresponding author upon request.

\section{Conflicts of Interest}

No potential conflicts of interest relevant to this article were reported.

\section{Acknowledgments}

Macrogen Inc. (Seoul, Korea) provided technical services for genetic analysis. The results reported here were based partly upon data generated by TCGA and European Bioinformatics Institute. This study was funded by Hallym University Research (HURF-2017-41).

\section{Supplementary Materials}

Supplemental Table 1: the top 50 features of Gene Set Enrichment Analysis based on metastasis in pheochromocytoma/paraganglioma from TCGA and COMETE cohort. Supplemental Table 2: the common differentially expressed (up/downregulated) genes (DEGs) extracted from TCGA and COMETE cohort. Supplemental Table 3: the 34 common differentially expressed (up/downregulated) genes (DEGs) validated in GSE67066 cohort. (Supplementary Materials)

\section{References}

[1] J. W. M. Lenders, G. Eisenhofer, M. Mannelli, and K. Pacak, "Phaeochromocytoma," The Lancet, vol. 366, no. 9486, pp. 665$675,2005$.

[2] E. P. Corssmit and J. A. Romijn, "Clinical management of paragangliomas," European Journal of Endocrinology, vol. 171, no. 6, pp. R231-R243, 2014.

[3] C. M. Kiernan and C. C. Solórzano, "Pheochromocytoma and paraganglioma: diagnosis, genetics, and treatment," Surgical Oncology Clinics of North America, vol. 25, no. 1, pp. 119-138, 2016.

[4] K. E. Joynt, J. J. Moslehi, and K. L. Baughman, "Paragangliomas: etiology, presentation, and management," Cardiology in Review, vol. 17, no. 4, pp. 159-164, 2009.

[5] E. L. Bravo and R. Tagle, "Pheochromocytoma: State-of-the-art and future prospects," Endocrine Reviews, vol. 24, no. 4, pp. 539553, 2003.

[6] J. W. M. Lenders, Q.-Y. Duh, and G. Eisenhofer, "Pheochromocytoma and paraganglioma : an endocrine society clinical practice guideline," The Journal of Clinical Endocrinology \& Metabolism, vol. 99, no. 6, pp. 1915-1942, 2014.

[7] A. Chrisoulidou, G. Kaltsas, I. Ilias, and A. B. Grossman, "The diagnosis and management of malignant phaeochromocytoma and paraganglioma," Endocrine-Related Cancer, vol. 14, no. 3, pp. 569-585, 2007.

[8] E. Baudin, M. A. Habra, F. Deschamps et al., "Therapy of endocrine disease: treatment of malignant pheochromocytoma and paraganglioma," European Journal of Endocrinology, vol. 171, no. 3, pp. R111-R122, 2014.

[9] R. Adjallé, P. F. Plouin, K. Pacak, and H. Lehnert, “Treatment of malignant pheochromocytoma," Hormone and Metabolic Research, vol. 41, no. 9, pp. 687-696, 2009.

[10] A. S. Tischler, K. Pacak, and G. Eisenhofer, "The adrenal medulla and extra-adrenal paraganglia: Then and now," Endocrine Pathology, vol. 25, no. 1, pp. 49-58, 2014.

[11] E. E. Elder, G. Elder, and C. Larsson, "Pheochromocytoma and functional paraganglioma syndrome: No longer the $10 \%$ tumor," Journal of Surgical Oncology, vol. 89, no. 3, pp. 193-201, 2005.

[12] K. F. Andersen, R. Altaf, A. Krarup-Hansen et al., "Malignant pheochromocytomas and paragangliomas - The importance of 
a multidisciplinary approach," Cancer Treatment Reviews, vol. 37, no. 2, pp. 111-119, 2011.

[13] L. D. R. Thompson, "Pheochromocytoma of the adrenal gland scaled score (PASS) to separate benign from malignant neoplasms: a clinicopathologic and immunophenotypic study of 100 cases," The American Journal of Surgical Pathology, vol. 26, no. 5, pp. 551-566, 2002.

[14] P. Goffredo, J. A. Sosa, and S. A. Roman, "Malignant pheochromocytoma and paraganglioma: A population level analysis of long-term survival over two decades," Journal of Surgical Oncology, vol. 107, no. 6, pp. 659-664, 2013.

[15] O. Gimm, C. DeMicco, A. Perren, F. Giammarile, M. K. Walz, and L. Brunaud, "Malignant pheochromocytomas and paragangliomas: A diagnostic challenge," Langenbeck's Archives of Surgery, vol. 397, no. 2, pp. 155-177, 2012.

[16] R. R. De Krijger, F. H. Van Nederveen, E. Korpershoek, and W. N. M. Dinjens, "New developments in the detection of the clinical behavior of pheochromocytomas and paragangliomas," Endocrine Pathology, vol. 17, no. 2, pp. 137-141, 2006.

[17] T. Scholz, C. Schulz, S. Klose, and H. Lehnert, "Diagnostic management of benign and malignant pheochromocytoma," Experimental and Clinical Endocrinology \& Diabetes, vol. 115, no. 03, pp. 155-159, 2007.

[18] P. Björklund, K. Pacak, and J. Crona, "Precision medicine in pheochromocytoma and paraganglioma: current and future concepts," Journal of Internal Medicine, vol. 280, no. 6, pp. 559$573,2016$.

[19] F. Feng, Y. Zhu, X. Wang et al., "Predictive factors for malignant pheochromocytoma: analysis of 136 patients," The Journal of Urology, vol. 185, no. 5, pp. 1583-1589, 2011.

[20] L. Fishbein, I. Leshchiner, and V. Walter, "Comprehensive molecular characterization of pheochromocytoma and paraganglioma," Cancer Cell, vol. 31, no. 2, pp. 181-193, 2017.

[21] L. J. Castro-Vega, E. Letouzé, N. Burnichon et al., "Multi-omics analysis defines core genomic alterations in pheochromocytomas and paragangliomas," Nature Communications, vol. 6, no. 1, 2015.

[22] N. Burnichon, L. Vescovo, L. Amar et al., "Integrative genomic analysis reveals somatic mutations in pheochromocytoma and paraganglioma," Human Molecular Genetics, vol. 20, no. 20, Article ID ddr324, pp. 3974-3985, 2011.

[23] L. Evenepoel, F. H. Van Nederveen, L. Oudijk et al., "Expression of contactin 4 is associated with malignant behavior in pheochromocytomas and paragangliomas," The Journal of Clinical Endocrinology \& Metabolism, vol. 103, no. 1, pp. 46-55, 2018.

[24] D. S. Chandrashekar, B. Bashel, S. A. H. Balasubramanya et al., "UALCAN: a portal for facilitating tumor subgroup gene expression and survival analyses," Neoplasia (United States), vol. 19, no. 8, pp. 649-658, 2017.

[25] O. Hamidi, W. F. Young, N. M. Iñiguez-Ariza et al., "Malignant pheochromocytoma and paraganglioma: 272 patients over 55 years," The Journal of Clinical Endocrinology \& Metabolism, vol. 102, no. 9, pp. 3296-3305, 2017.

[26] Y. M. Choi, T.-Y. Sung, W. G. Kim et al., "Clinical course and prognostic factors in patients with malignant pheochromocytoma and paraganglioma: A single institution experience," Journal of Surgical Oncology, vol. 112, no. 8, pp. 815-821, 2015.

[27] M. Ayala-Ramirez, L. Feng, M. M. Johnson et al., "Clinical risk factors for malignancy and overall survival in patients with pheochromocytomas and sympathetic paragangliomas: primary tumor size and primary tumor location as prognostic indicators," The Journal of Clinical Endocrinology \& Metabolism, vol. 96, no. 3, pp. 717-725, 2011.

[28] A. S. Tischler and R. R. De Krijger, "Pathology of pheochromocytoma and paraganglioma," Endocrine-Related Cancer, vol. 22, no. 4, pp. T123-T133, 2015.

[29] N. Kimura, T. Watanabe, T. Noshiro, S. Shizawa, and Y. Miura, "Histological grading of adrenal and extra-adrenal pheochromocytomas and relationship to prognosis: A clinicopathological analysis of 116 adrenal pheochromocytomas and 30 extraadrenal sympathetic paragangliomas including 38 malignant tumors," Endocrine Pathology, vol. 16, no. 1, pp. 23-32, 2005.

[30] G. Eisenhofer and A. S. Tischler, "Neuroendocrine cancer: Closing the GAPP on predicting metastases," Nature Reviews Endocrinology, vol. 10, no. 6, pp. 315-316, 2014.

[31] I. Suh, D. Shibru, G. Eisenhofer et al., "Candidate genes associated with malignant pheochromocytomas by genomewide expression profiling," Annals of Surgery, vol. 250, no. 6, pp. 983-990, 2009.

[32] J. Favier, L. Amar, and A.-P. Gimenez-Roqueplo, "Paraganglioma and phaeochromocytoma: from genetics to personalized medicine," Nature Reviews Endocrinology, vol. 11, no. 2, pp. 101-111, 2015.

[33] L. Santarpia, M. A. Habra, and C. Jiménez, "Malignant pheochromocytomas and paragangliomas: Molecular signaling pathways and emerging therapies," Hormone and Metabolic Research, vol. 41, no. 9, pp. 680-686, 2009.

[34] F. Khatami, M. Mohammadamoli, and S. M. Tavangar, "Genetic and epigenetic differences of benign and malignant pheochromocytomas and paragangliomas (PPGLs)," Endocrine Regulations, vol. 52, no. 1, pp. 41-54, 2018.

[35] J. Favier and A.-P. Gimenez-Roqueplo, "Pheochromocytomas: the (pseudo)-hypoxia hypothesis," Best Practice \& Research Clinical Endocrinology \& Metabolism, vol. 24, no. 6, pp. 957-968, 2010.

[36] H. M. Rossitti, P. Söderkvist, and O. Gimm, "Extent of surgery for phaeochromocytomas in the genomic era," British Journal of Surgery, vol. 105, no. 2, pp. e84-e98, 2018.

[37] A.-P. Gimenez-Roqueplo, P. L. Dahia, and M. Robledo, "An update on the genetics of paraganglioma, pheochromocytoma, and associated hereditary syndromes," Hormone and Metabolic Research, vol. 44, no. 5, pp. 328-333, 2012.

[38] S. O. Zhikrivetskaya, A. V. Snezhkina, A. R. Zaretsky et al., "Molecular markers of paragangliomas/pheochromocytomas," Oncotarget, vol. 8, no. 15, pp. 25756-25782, 2017.

[39] S. Fan, Y. Wang, Z. Zhang et al., "High expression of glutamateammonia ligase is associated with unfavorable prognosis in patients with ovarian cancer," Journal of Cellular Biochemistry, vol. 119, no. 7, pp. 6008-6015, 2018.

[40] L. Amar, E. Baudin, N. Burnichon et al., "Succinate dehydrogenase $B$ gene mutations predict survival in patients with malignant pheochromocytomas or paragangliomas," The Journal of Clinical Endocrinology \& Metabolism, vol. 92, no. 10, pp. 38223828, 2007.

[41] L. J. Castro-Vega, A. Buffet, A. A. De Cubas et al., "Germline mutations in FH confer predisposition to malignant pheochromocytomas and paragangliomas," Human Molecular Genetics, vol. 23, no. 9, Article ID ddt639, pp. 2440-2446, 2014.

[42] B. Bausch, F. Schiavi, Y. Ni et al., "Clinical characterization of the pheochromocytoma and paraganglioma susceptibility genes SDHA, TMEM127, MAX, and SDHAF2 for gene-informed prevention," JAMA Oncology, vol. 3, no. 9, pp. 1204-1212, 2017. 
[43] A. Buffet, A. Morin, L.-J. Castro-Vega et al., "Germline mutations in the mitochondrial 2-oxoglutarate/malate carrier SLC25A11 gene confer a predisposition to metastatic paragangliomas," Cancer Research, vol. 78, no. 8, pp. 1914-1922, 2018.

[44] J. Turchini, V. K. Y. Cheung, A. S. Tischler, R. R. De Krijger, and A. J. Gill, "Pathology and genetics of phaeochromocytoma and paraganglioma," Histopathology, vol. 72, no. 1, pp. 97-105, 2018.

[45] E. Letouzé, C. Martinelli, C. Loriot et al., "SDH mutations establish a hypermethylator phenotype in paraganglioma," Cancer Cell, vol. 23, no. 6, pp. 739-752, 2013.

[46] A. A. De Cubas, E. Korpershoek, L. Inglada-Pérez et al., "DNA methylation profiling in pheochromocytoma and paraganglioma reveals diagnostic and prognostic markers," Clinical Cancer Research, vol. 21, no. 13, pp. 3020-3030, 2015.

[47] R. Simon, "Roadmap for developing and validating therapeutically relevant genomic classifiers," Journal of Clinical Oncology, vol. 23, no. 29, pp. 7332-7341, 2005. 


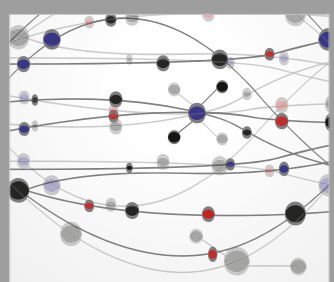

The Scientific World Journal
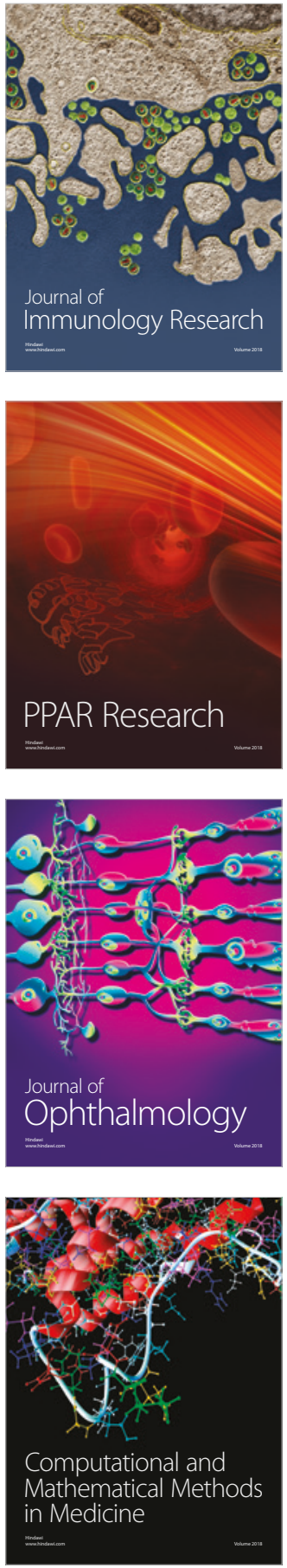

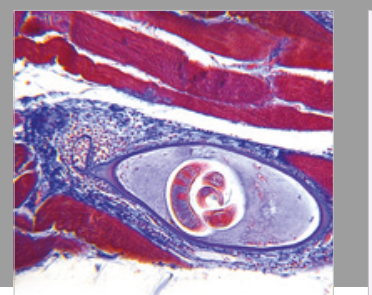

Gastroenterology Research and Practice

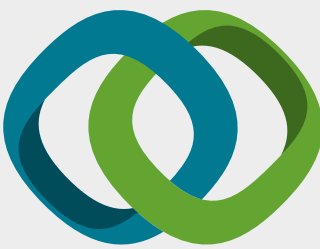

\section{Hindawi}

Submit your manuscripts at

www.hindawi.com
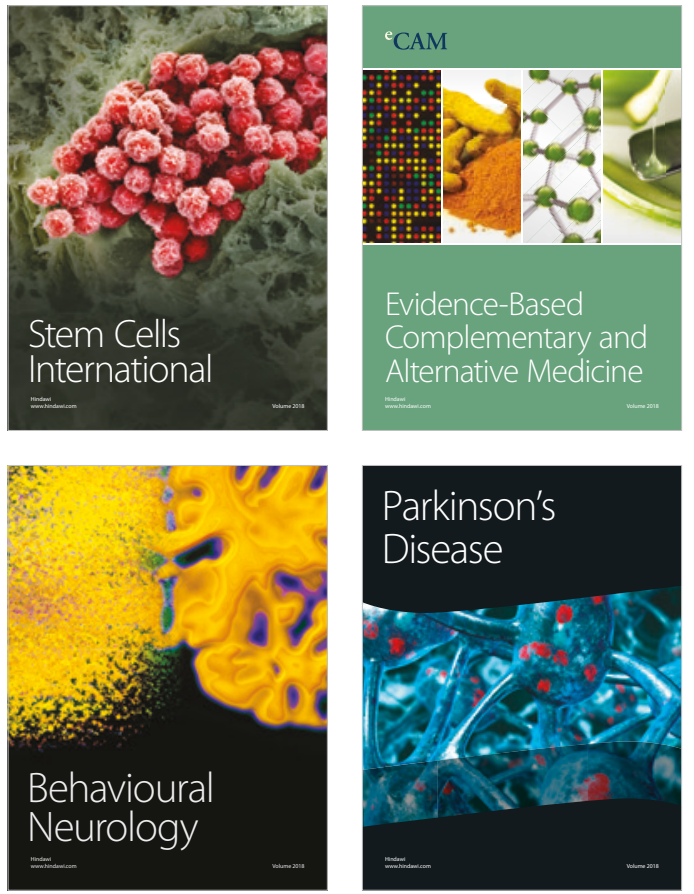

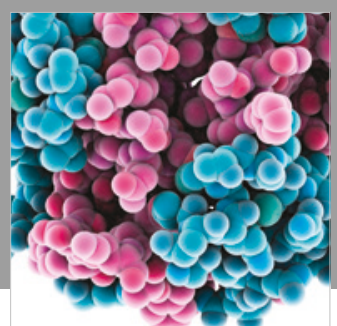

ournal of

Diabetes Research

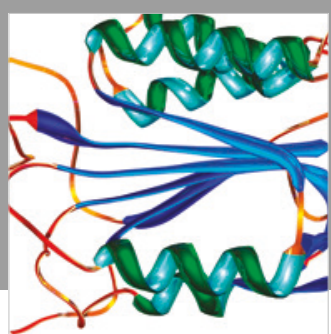

Disease Markers
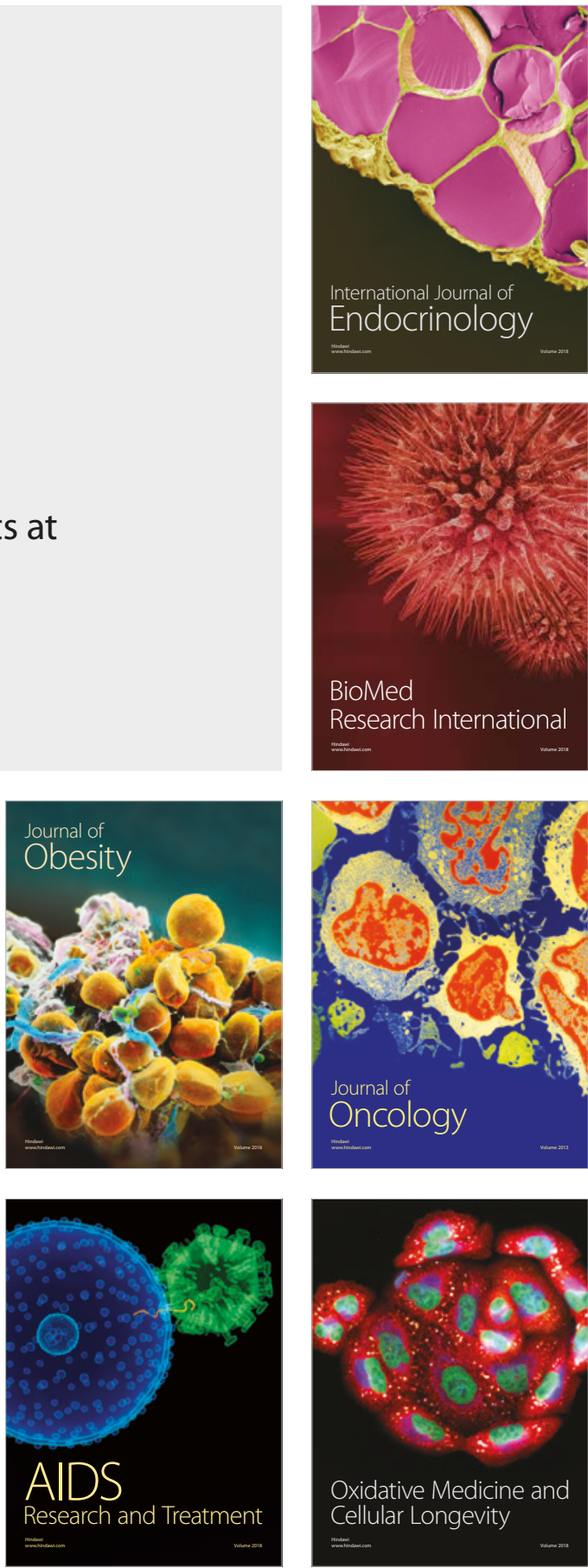